TRANSACTIONS OF THE

AMERICAN MATHEMATICAL SOCIETY

Volume 363, Number 11, November 2011, Pages 5721-5744

S 0002-9947(2011)05280-3

Article electronically published on June 17, 2011

\title{
NORMALITY AND REPELLING PERIODIC POINTS
}

\author{
JIANMING CHANG AND LAWRENCE ZALCMAN
}

\begin{abstract}
Let $k \geq 3(\geq 2)$ be an integer and $\mathcal{F}$ be a family of functions meromorphic in a domain $D \subset \mathbb{C}$, all of whose poles have multiplicity at least 2 (at least 3). If in $D$ each $f \in \mathcal{F}$ has neither repelling fixed points nor repelling periodic points of period $k$, then $\mathcal{F}$ is a normal family in $D$. Examples are given to show that the conditions on poles are necessary and sharp.
\end{abstract}

\section{INTRODUCTION AND MAIN RESULTS}

A family $\mathcal{F}$ of meromorphic functions defined in a plane domain $D \subset \mathbb{C}$ is said to be normal in $D$ if each sequence $\left\{f_{n}\right\} \subset \mathcal{F}$ contains a subsequence which converges spherically locally uniformly in $D$ to a meromorphic function or $\infty$; see [16, 20, 24.

In recent years, there have been many interesting results on normal families of holomorphic or meromorphic functions defined by conditions on fixed points or periodic points. This subject starts from a problem of L. Yang [23, Problem 8]. To state this problem and related results, we require the following notation and definitions.

Let $f: D \rightarrow \overline{\mathbb{C}}$ be a meromorphic function. Then the iterates $f^{n}: D_{n} \rightarrow \overline{\mathbb{C}}$ of $f$ are defined inductively by $D_{1}=D, f^{1}=f$ and

$$
D_{n}=f^{-1}\left(D_{n-1}\right)=\left\{z \in D: f(z) \in D_{n-1}\right\}, \quad f^{n}=f^{n-1} \circ f \text { for } n \geq 2 .
$$

Note that $D_{n+1} \subset D_{n} \subset D$ for all $n \in \mathbb{N}$. See [2, 3, 11, 14, 15].

Let $z_{0} \in D$. If there exists a smallest integer $p \in \mathbb{N}$ such that $z_{0} \in D_{p}, f^{p}\left(z_{0}\right)=$ $z_{0}$, then $z_{0}$ is said to be a periodic point of period $p$ of $f$ and the corresponding cycle $\left\{z_{0}, f\left(z_{0}\right), \cdots, f^{p-1}\left(z_{0}\right)\right\}$ is said to be a periodic cycle of period $p$ of $f$ in $D$. A periodic point of period 1 is said to be a fixed point. Define the multiplier of the periodic point $z_{0}$ (and the corresponding cycle) by $\lambda=\left(f^{p}\right)^{\prime}\left(z_{0}\right)$. According to $|\lambda|<1,|\lambda|=1$, or $|\lambda|>1$, the periodic point $z_{0}$ (and the corresponding cycle) is said to be attracting, neutral, or repelling. If $|\lambda|=1$, then according to whether there is some integer $m$ such that $(\lambda)^{m}=1$ or not, $z_{0}$ is said to be rationally neutral or irrationally neutral. A fixed point which is either repelling or has multiplier 1 is said to be weakly repelling; see [2, 3, 4, 7, 8, 14, 22.

Received by the editors September 6, 2009.

2000 Mathematics Subject Classification. Primary 30D45, 30D05, 37F10, 37C25.

Key words and phrases. Meromorphic function, normal family, iterate, fixed point, periodic point.

The research of the first author was supported by NNSF of China (Grant No. 10871094), NSFU of Jiangsu, China (Grant No. 08KJB110001), Qinglan Project of Jiangsu, China, and the SRF for ROCS, SEM..

The research of the second author was supported by Israel Science Foundation Grant 395/07. This work is part of the European Science Foundation Networking Programme HCAA..

(C)2011 American Mathematical Society Reverts to public domain 28 years from publication 
The problem of Yang mentioned above can be stated as follows.

Problem 1. Let $\mathcal{F}$ be a family of entire functions and $D \subset \mathbb{C}$ be a domain. If there exists an integer $k \geq 2$ such that each $f \in \mathcal{F}$ and its $k$-th iterate $f^{k}$ has no fixed points in $D$, must $\mathcal{F}$ be normal in $D$ ?

Essén and $\mathrm{Wu}$ [14, 15] answered Problem 1 affirmatively with the following more general result.

Theorem A. Let $\mathcal{F}$ be a family of functions holomorphic in $D$. If for each $f \in \mathcal{F}$ there exists an integer $k=k(f) \geq 2$ such that the $k$-th iterate $f^{k}$ has no repelling fixed points in $D$, then $\mathcal{F}$ is normal in $D$.

The following result [10] is a generalization of Theorem A, in which only the fixed points and the periodic points of period $k$ of $f \in \mathcal{F}$ are considered. We remark that every periodic point of period $j$ of $f$ with $j$ a divisor of $k$ is a fixed point of the $k$-th iterate $f^{k}$, and vice versa.

Theorem B. Let $K<\infty$ be a positive number, $D \subset \mathbb{C}$ be a domain, and $\mathcal{F}$ be a family of functions holomorphic in $D$. If for every $f \in \mathcal{F},\left|(f)^{\prime}(\eta)\right| \leq K$ for every fixed point $\eta$ of $f$ in $D$ and there exists a positive integer $k=k(f)$ such that $f$ has no repelling periodic points of period $k$ in $D$, then $\mathcal{F}$ is normal in $D$, provided that one of the following conditions holds:

(a) $K<3$ and $k \geq 2$ for all $f \in \mathcal{F}$;

(b) $K<2 \sqrt{2}+1$ and $k \geq 3$ for all $f \in \mathcal{F}$;

(c) $K<\infty$ and $k \geq 4$ for all $f \in \mathcal{F}$.

Thus it is natural to study the following problem for families of meromorphic functions [11].

Problem 2. Let $\mathcal{F}$ be a family of functions meromorphic in a domain $D \subset \mathbb{C}$. If there exists an integer $k \geq 2$ such that for each $f \in \mathcal{F}$ the $k$-th iterate $f^{k}$ has no repelling fixed points in $D$, must $\mathcal{F}$ be normal in $D$ ?

The family $\{1 /(n z)\}$, which is not normal at $z=0$, shows that the answer to Problem 2 is negative [11, Example 1]. However, we have proved the following result [13, Theorem 2].

Theorem C. Let $\mathcal{F}$ be a family of functions meromorphic in a domain $D \subset \mathbb{C}$ and $\delta<1$ be a positive number. If there exists an integer $k \geq 2$ such that for each $f \in \mathcal{F}$ all the fixed points $\eta \in D$ of the $k$-th iterate $f^{k}$ satisfy $\left|\left(f^{k}\right)^{\prime}(\eta)\right| \leq \delta$, then $\mathcal{F}$ is normal in $D$.

The condition in Theorem $\mathrm{C}$, that the fixed points of $f^{k}$ for all $f \in \mathcal{F}$ are uniformly attracting, is necessary and cannot be replaced by assuming that the fixed points of $f^{k}$ for all $f \in \mathcal{F}$ are attracting [13, Theorem 1].

Here, we continue to study Problem 2. We show that under some appropriate additional conditions, the answer to Problem 2 is positive.

Theorem 1. Let $k \geq 3$ be an integer and $\mathcal{F}$ be a family of meromorphic functions in $D$ such that each function in $\mathcal{F}$ has neither repelling fixed points nor repelling periodic points of period $k$ in $D$. If for each $f \in \mathcal{F}$ there exists a constant $a=$ $a(f) \in \overline{\mathbb{C}} \backslash D$ such that all a-points of $f$ in $D$ have multiplicity at least 2 , then $\mathcal{F}$ is normal in $D$. 
Since $\infty \in \overline{\mathbb{C}} \backslash D$, we have the following corollary.

Corollary 2. Let $k \geq 3$ be an integer and $\mathcal{F}$ be a family of meromorphic functions in $D$ such that each function in $\mathcal{F}$ has neither repelling fixed points nor repelling periodic points of period $k$ in $D$. If for each $f \in \mathcal{F}$ all poles of $f$ in $D$ have multiplicity at least 2 , then $\mathcal{F}$ is normal in $D$.

The example $\{1 /(n z)\}$ shows that the condition on the poles is necessary in Corollary 2 and that the constants $a$ cannot be in $D$ in Theorem 1 as $1 /(n z) \neq 0$ for all $n$. The following example shows that Corollary 2 (and Theorem 1 ) does not hold for $k=2$.

Example 1. Let

$$
\mathcal{F}=\left\{f_{n}(z)=\frac{z}{3}+\frac{2}{3 n^{3} z^{2}}: n=1,2,3, \cdots\right\} .
$$

Then each $f_{n}$ has a single double pole and has neither repelling fixed points nor (repelling) periodic points of period 2 in $\mathbb{C}$, since

$$
f_{n}(z)=z-\frac{2\left(z^{3}-1 / n^{3}\right)}{3 z^{2}}, \quad f_{n}^{2}(z)=z-\frac{8\left(z^{3}-1 / n^{3}\right)^{3}}{9 z^{2}\left(z^{3}+2 / n^{3}\right)^{2}} .
$$

However, we have $f_{n}(0)=\infty$ and $f_{n}(1 / n)=1 / n \rightarrow 0$ as $n \rightarrow \infty$. It follows that the family $\mathcal{F}=\left\{f_{n}\right\}$ is not equi-continuous. Hence $\mathcal{F}$ is not normal at $z=0$.

For $k=2$, we have

Theorem 3. Let $\mathcal{F}$ be a family of meromorphic functions in $D$ such that for each function $f \in \mathcal{F}, f^{2}$ has no repelling fixed points in $D$. If for every $f \in \mathcal{F}$ there exists a constant $a=a(f) \in \overline{\mathbb{C}} \backslash D$ such that all a-points of $f$ in $D$ have multiplicity at least 3 , then $\mathcal{F}$ is normal in $D$.

Corollary 4. Let $\mathcal{F}$ be a family of meromorphic functions in $D$ such that for each function $f \in \mathcal{F}, f^{2}$ has no repelling fixed points in $D$. If for every $f \in \mathcal{F}$ all poles of $f$ in $D$ have multiplicity at least 3 , then $\mathcal{F}$ is normal in $D$.

We also have the following result which is a generalization of Theorem $\mathrm{C}$.

Theorem 5. Let $k \geq 2$ be an integer and $\mathcal{F}$ a family of functions meromorphic in $D \subset \mathbb{C}$ having no repelling periodic points of period $k$ in $D$. If there exists a positive number $\delta<1$ such that for each $f \in \mathcal{F},\left|f^{\prime}(z)\right| \leq \delta$ whenever $z$ is a fixed point of $f$ in $D$, then $\mathcal{F}$ is normal in $D$.

The plan of this paper is as follows. In Section 2, we state and prove a number of auxiliary results, some of which are of independent interest. In Section 3, we give the proofs of theorems.

\section{Auxiliary Results}

In this section, we state some known results and prove the main lemmas that are required in the proofs of our results.

Lemma 1 ([3, Theorem 5]). Let $f$ be a transcendental meromorphic function and $k \geq 2$ a positive integer. Then $f$ has infinitely many repelling periodic points of period $k$ in $\mathbb{C}$. 
This result, which answers a problem of Baker, does not hold for rational functions of degree at least 2. Baker [1] proved that there are rational functions of degree at least 2 which have no periodic point of period $k=2$ or $k=3$. For rational functions, we proved

Lemma 2 ([12, Theorem 2]). Let $R$ be a rational function of degree $d \geq 2$ and let $k \geq 2$ be an integer. Denote by $N_{r p}(k)$ the number of repelling periodic points of period $k$ of $R$. Then

$$
N_{r p}(k) \geq d^{k}-\sum_{j \mid k, j<k} d^{j}-4 k(d-1) .
$$

As a corollary to Lemma 2, we have

Lemma 3. Let $R$ be a rational function of degree $\geq 2$ and let $k \geq 5$ be an integer. Then $R$ has at least two repelling periodic cycles of period $k$, and hence at least one of them lies in $\mathbb{C}$.

Proof. Suppose that $R$ has at most one repelling periodic cycle of period $k$. Then $N_{r p}(k) \leq k$. Thus, by Lemma 2 ,

$$
f_{k}(d):=d^{k}-\sum_{j \mid k, j<k} d^{j}-4 k(d-1)-k \leq 0 .
$$

Let $m$ be the largest integer less than $k$ that divides $k$. Then $m \leq k / 2$. Note that $k \geq 5$ and $d \geq 2$, so that $d^{k / 2}-1>d^{2}-1 \geq 3(d-1) \geq 3$. Thus

$$
\begin{aligned}
d^{k} & \leq \sum_{j \mid k, j<k} d^{j}+4 k(d-1)+k \leq \sum_{j=1}^{m} d^{j}+5 k(d-1) \\
& =\frac{d}{d-1}\left(d^{m}-1\right)+5 k(d-1)<\left(2+\frac{5 k}{3}\right)\left(d^{k / 2}-1\right) \\
& \leq \frac{31 k}{15}\left(d^{k / 2}-1\right)<\frac{31 k}{15} d^{k / 2}
\end{aligned}
$$

so that

$$
d<\left(\frac{31 k}{15}\right)^{2 / k}
$$

Let $\phi(x)=(31 x / 15)^{2 / x}$. Then for $x>2$,

$$
\phi^{\prime}(x)=\frac{2}{x^{2}}\left(\frac{31 x}{15}\right)^{2 / x}\left(1-\log \frac{31 x}{15}\right)<0 .
$$

Thus $\phi(x)$ is decreasing for $x>2$. It follows from (2) that $d<\phi(k) \leq \phi(5)=$ $(31 \times 5 / 15)^{2 / 5}<3$. Moreover, if $k \geq 9$, then $d<\phi(k) \leq \phi(9)=(31 \times 9 / 15)^{2 / 9}<2$. Hence $d=2$ and $5 \leq k \leq 8$. However, by direct calculations we have $f_{5}(2)=5$, $f_{6}(2)=20, f_{7}(2)=91, f_{8}(2)=194$. This contradicts (1). The lemma is proved.

It is interesting that Lemma 3 does not hold for $k \leq 4$.

Example 2 ([1, Example 1]). Let

$$
R(z)=z+\frac{(-2+\sqrt{2} i)\left(3 z^{2}+2-2 \sqrt{2} i\right)}{6 z} .
$$


Then

$$
\begin{aligned}
& R^{2}(z)=z-\frac{3 z^{4}+4 z^{2}+4}{2 z\left(z^{2}+2\right)} \text { and } \\
& R^{4}(z)=z-\frac{\left(3 z^{4}+4 z^{2}+4\right)\left(z^{4}+4 z^{2}-4\right)^{3}}{4 z\left(z^{2}+2\right)\left(z^{2}+2 z+2\right)\left(z^{2}-2 z+2\right)\left(z^{8}+8 z^{6}+40 z^{4}+32 z^{2}+16\right)} .
\end{aligned}
$$

Thus $R$ has no repelling periodic cycle of period 4 .

However, for $k \leq 4$ we have the following four results, which are proved below.

Lemma 4. Let $R$ be a rational function of degree at least 2 . Then $R$ has either a repelling fixed point in $\mathbb{C}$ or a repelling periodic cycle of period 4 in $\mathbb{C}$.

Lemma 5. Let $R$ be a rational function of degree at least 2 . Then $R$ has either a repelling fixed point in $\mathbb{C}$ or a repelling periodic cycle of period 3 in $\mathbb{C}$, unless $R$ is affinely conjugate to one of the functions

$$
\begin{aligned}
& z-\frac{3 z^{2}}{2(z-1)}, z+\frac{(-3 \pm \sqrt{3} i) z(z-1)}{2(2 z-1)}, \text { or } \\
& z-\frac{c\left(z-z_{0}\right)^{2}\left[z_{0}\left(z-z_{0}\right)+1\right]}{c z_{0}\left(z-z_{0}\right)^{2}+\left(c+z_{0}+1\right)\left(z-z_{0}\right)+1},
\end{aligned}
$$

where the constants $c, z_{0}$ satisfy $c^{2}+3 c+3=0$ and $z_{0}^{3}+(4 c+6) z_{0}^{2}+2 c z_{0}-2=0$.

Here and in the sequel, for two rational functions $U$ and $V$, we say that $U$ is affinely conjugate to $V$ if there exist constants $a(\neq 0)$ and $b$ such that $a U(z)+b \equiv$ $V(a z+b)$; see [1].

Lemma 6. Let $R$ be a rational function of degree at least 2 such that $R$ has no pole with multiplicity $\leq 2$. Then $R$ has either a repelling fixed point in $\mathbb{C}$ or a repelling periodic cycle of period 2 in $\mathbb{C}$.

Lemma 7. Let $R$ be a rational function of degree at least 2 such that $R$ has no fixed point in $\mathbb{C}$ with multiplier 1 or -1 . Then $R$ has either a repelling fixed point in $\mathbb{C}$ or a repelling periodic cycle of period 2 in $\mathbb{C}$.

To prove Lemmas $4-7$, we require the following results (Lemmas 8-15) from complex dynamics.

Lemma 8 ([17, Corollary 12.7]; cf. [21, Lemma 25]). Let $R$ be a rational function of degree $\geq 2$. Then $R$ has a weakly repelling fixed point in $\overline{\mathbb{C}}$.

Now let $\left\{z_{0}, R\left(z_{0}\right), \cdots, R^{p-1}\left(z_{0}\right)\right\}$ be an attracting periodic cycle of period $p$ of $R$. Then the Fatou set of $R$ has $p$ components $U_{j}(0 \leq j \leq p-1)$ such that $R^{j}\left(z_{0}\right) \in U_{j}$ and $R^{n p}(z) \rightarrow R^{j}\left(z_{0}\right)$ in $U_{j}$ as $n \rightarrow \infty$. The union $\bigcup_{j=0}^{p-1} U_{j}$ is called the immediate basin of attraction associated to the attracting periodic cycle $\left\{z_{0}, R\left(z_{0}\right), \cdots, R^{p-1}\left(z_{0}\right)\right\}$; see [8, p. 58].

Lemma 9 ([8, p. 59, Theorem 2.2]). The immediate basin of attraction associated to an attracting periodic cycle contains at least one critical point.

Here and in the sequel, a point $z_{0} \in \mathbb{C}$ is called a critical point of $R$ (of multiplicity $p$ ) if $z_{0}$ is a zero of $R^{\prime}$ (of multiplicity $p$ ) or a multiple pole of $R$ (of multiplicity $p+1$ ); $\infty$ is a critical point of $R$ (of multiplicity $p$ ) if 0 is a critical point of $g$ (of 
multiplicity $p$ ), where $g(z)=R(1 / z)$. A useful fact is that a rational function of degree $d \geq 2$ has at most $2 d-2$ critical points counting multiplicity; see [8, p. 54].

Now let $\left\{z_{0}, R\left(z_{0}\right), \cdots, R^{p-1}\left(z_{0}\right)\right\} \subset \mathbb{C}$ be a rationally neutral cycle of period $p$. Then there exists a smallest integer $m \geq 1$ such that $\left[\left(R^{p}\right)^{\prime}\left(z_{0}\right)\right]^{m}=1$. Hence there exists a constant $c \neq 0$ and a positive integer $k$ such that near $z_{0}$

$$
R^{p m}(z)=z+c\left(z-z_{0}\right)^{k m+1}[1+o(1)]
$$

see [8, p. 41] and [6, p. 8]. Furthermore, for any $n \in \mathbb{N}$,

$$
R^{n p m}(z)=z+n c\left(z-z_{0}\right)^{k m+1}[1+o(1)] .
$$

According to the Leau-Fatou petal theorem (see [22, p. 75], the Flower Theorem), for each $0 \leq j \leq p-1$, the Fatou set of $R$ has $k m$ components $U_{j, i}(1 \leq i \leq k m)$ such that $R^{j}\left(z_{0}\right) \in \partial U_{j, i}$; in $U_{j, i}, R^{n p}(z) \rightarrow R^{j}\left(z_{0}\right),(n \rightarrow \infty)$. These $U_{j, i}$ are called Leau domains or attracting petals. They can be divided into $k$ groups, where each group $G$ has $p m$ Leau domains such that $R(G)=G$. That is, each group $G$ can be written as $G=\left\{R^{j}(U), 0 \leq j \leq p m-1\right\}\left(R^{p m}(U)=U\right)$. The group $G$ is called a cycle of Leau domains associated to the rationally neutral cycle $\left\{z_{0}, R\left(z_{0}\right), \cdots, R^{p-1}\left(z_{0}\right)\right\}$. The union $\bigcup_{j=0}^{p m-1} R^{j}(U)$ is called the immediate basin of attraction associated to a rationally neutral cycle $\left\{z_{0}, R\left(z_{0}\right), \cdots, R^{p-1}\left(z_{0}\right)\right\}$. See [6, p. 8], [8, p. 60] and [22, pp. $72-77]$.

By conjugation, one can define the cycles of Leau domains or immediate basins of attraction associated to a rationally neutral cycle of period $p$ containing $\infty$.

Lemma 10 ([8, p. 60, Theorem 2.3]). Each immediate basin of attraction associated to a rationally neutral periodic cycle contains a critical point.

The relation between critical points and irrationally neutral periodic cycles is more complicated. Using quasi-conformal surgery, Shishikura [21, Proposition 1] proved that for a rational function $R$ of degree $d \geq 2$, the number of critical points (ignoring multiplicity) of $R$ contained in the Fatou set but not in the inverse images of Herman rings plus the number of irrationally neutral periodic cycles of $R$ does not exceed the number of critical points (ignoring multiplicity) of $R$. As a corollary, we have

Lemma 11 (21, Proposition 1]). For a rational function $R$ of degree $d \geq 2$, the number of critical points (ignoring multiplicity) of $R$ contained in the immediate basins of attraction associated to the attracting periodic cycles and rationally neutral cycles plus the number of irrationally neutral periodic cycles of $R$ does not exceed the number of critical points (ignoring multiplicity) of $R$, and hence is at most $2 d-2$.

By Lemmas 9-11, we have

Lemma 12 ([21, Corollary 1]). Let $R$ be a rational function of degree $\geq 2$. Then $R$ has at most $2 d-2$ non-repelling periodic cycles.

Lemma 13 ([12, Lemma 4]). Let $R$ be a rational function of degree $d \geq 2$ such that $\infty$ is a weakly repelling fixed point of $R$. Then $R$ has the form

$$
R(z)=z+c \frac{Q(z)}{P(z)}
$$

where $c \neq 0$ is a constant and $P, Q$ are co-prime monic polynomials with degrees $p$ and $q$, respectively, such that $q \leq p+1=d$ and that $0<|c+1|<1$ when $q=p+1$. 
Furthermore, for $k \geq 2$,

$$
R^{k}(z)=z+c_{k} \frac{Q_{k}(z)}{P_{k}(z)}
$$

with constant $c_{k} \neq 0$ and co-prime monic polynomials $P_{k}$ and $Q_{k}$ satisfying $\operatorname{deg}\left(Q_{k}\right)$ $=q+(p+1)^{k}-(p+1)$.

Remark 1. When $q \leq p, \infty$ is a fixpoint with multiplier 1 , and near $z=0$,

$$
\frac{1}{R\left(\frac{1}{z}\right)}=z-c z^{p-q+2}[1+o(1)]
$$

It follows that there are $p-q+1$ cycles of Leau domains (each cycle consists of one Leau domain) associated to the fixed point $\infty$, so that by Lemma 11 there are at least $p-q+1$ critical points associated to the fixed point $\infty$.

The following lemma follows from the proof of Lemma 2; cf. the proof of Theorem 2 in 12 .

Lemma 14. Let $R$ be a rational function of degree $d \geq 2$ of the form (1) with constant $c \neq 0$ and co-prime monic polynomials $P, Q$ satisfying the properties stated in Lemma 13. Then the polynomial $Q_{k}$ in Lemma 13 has the following representation:

$$
Q_{k}(z)=\prod_{j \mid k}\left\{\prod_{i=1}^{m_{j}}\left[\prod_{\zeta \in \Gamma_{j, i}}(z-\zeta)\right]^{\nu_{j, i}^{(k)}+1} \prod_{i=m_{j}+1}^{n_{j}}\left[\prod_{\zeta \in \Gamma_{j, i}}(z-\zeta)\right]\right\}
$$

so that by Lemma 13 ,

$$
q+(p+1)^{k}-(p+1)=\sum_{j \mid k} \sum_{i=1}^{m_{j}} j \nu_{j, i}^{(k)}+\sum_{j \mid k} j n_{j} .
$$

Here $\Gamma_{j, i}(\subset \mathbb{C})$ are the periodic cycles of period $j, n_{j} \geq 0$ and $m_{j} \geq 0$ are the number of periodic cycles of period $j$ contained in $\mathbb{C}$ and the number of non-repelling periodic cycles of period $j$ contained in $\mathbb{C}$, respectively, and $\nu_{j, i}^{(k)} \geq 0$ are integers.

Remark 2. By (3) and (4), for $k_{1} \mid k_{2}$, if $\nu_{j, i}^{\left(k_{1}\right)}>0$, then $\nu_{j, i}^{\left(k_{2}\right)}=\nu_{j, i}^{\left(k_{1}\right)}$.

Remark 3. By Lemmas 9 and 10 (see the proof of Theorem 2 in [12]),

$$
\sum_{j \mid k} \sum_{i=1}^{m_{j}} j \nu_{j, i}^{(k)} \leq k N_{c}^{\prime}
$$

where $N_{c}^{\prime}$ is the number of critical points of $R$ (ignoring multiplicity) which lie in the Leau domains associated to the rationally neutral periodic cycles (of periods $j \mid k)$ contained in $\mathbb{C}$.

Remark 4 . Let $k \geq 2$ be a prime integer and set

$$
\begin{aligned}
& I=\left\{i: \nu_{1, i}^{(k)}=0\right\}, \quad I_{1}=\left\{i \in I: \quad \Gamma_{1, i} \text { is irrationally neutral }\right\}, \\
& J=\left\{i: \nu_{k, i}^{(k)}=0\right\}, \quad J_{1}=\left\{i \in J: \Gamma_{k, i} \text { is irrationally neutral }\right\} .
\end{aligned}
$$

Denote by $N_{c}^{\prime \prime}$ the number of critical points of $R$ (ignoring multiplicity) which lie in the the immediate basins of attraction associated to the attracting periodic 
cycles (of periods $j \mid k$ ) contained in $\mathbb{C}$, by $N_{c}$ the number of critical points of $R$ (ignoring multiplicity) in $\overline{\mathbb{C}}=\mathbb{C} \cup\{\infty\}$, and by $n_{\infty}$ the number of critical points of $R$ (ignoring multiplicity) which lie in the Leau domains associated to the rationally neutral fixed point $\infty$. By Remark 1 , we see that for $q=p+1, n_{\infty}=0$, while for $q \leq p, n_{\infty} \geq p-q+1$.

Then by Lemma 9 and Lemma 10, we have

$$
\sum_{i \in I \backslash I_{1}} 1+\sum_{i \in J \backslash J_{1}} 1+\sum_{i=1}^{m_{1}} \nu_{1, i}^{(1)}+\frac{1}{k} \sum_{1 \leq i \leq m_{1}, \nu_{1, i}^{(1)}=0} \nu_{1, i}^{(k)}+\sum_{i=1}^{m_{k}} \nu_{k, i}^{(k)} \leq N_{c}^{\prime}+N_{c}^{\prime \prime},
$$

and by Lemma 11,

$$
N_{c}^{\prime}+N_{c}^{\prime \prime}+n_{\infty}+\sum_{i \in I_{1}} 1+\sum_{i \in J_{1}} 1 \leq N_{c}
$$

Note that

$$
\begin{aligned}
& m_{1} \leq \sum_{i \in I} 1+\sum_{i=1}^{m_{1}} \nu_{1, i}^{(1)}+\frac{1}{k} \sum_{1 \leq i \leq m_{1}, \nu_{1, i}^{(1)}=0} \nu_{1, i}^{(k)} \\
& m_{k} \leq \sum_{i \in J} 1+\sum_{i=1}^{m_{k}} \nu_{k, i}^{(k)} .
\end{aligned}
$$

Thus, by (12)-(15),

$$
\begin{aligned}
& m_{1}+m_{k} \\
\leq & \sum_{i \in I} 1+\sum_{i \in J} 1+\sum_{i=1}^{m_{1}} \nu_{1, i}^{(1)}+\frac{1}{k} \sum_{1 \leq i \leq m_{1}, \nu_{1, i}^{(1)}=0} \nu_{1, i}^{(k)}+\sum_{i=1}^{m_{k}} \nu_{k, i}^{(k)} \\
\leq & N_{c}-n_{\infty},
\end{aligned}
$$

and by (9),

$$
\begin{gathered}
q=n_{1}+\sum_{i=1}^{m_{1}} \nu_{1, i}^{(1)}, \\
q+(p+1)^{k}-(p+1)=n_{1}+\sum_{i=1}^{m_{1}} \nu_{1, i}^{(k)}+k n_{k}+k \sum_{i=1}^{m_{k}} \nu_{k, i}^{(k)} .
\end{gathered}
$$

Thus

$$
(p+1)^{k}-(p+1)=k n_{k}+\sum_{1 \leq i \leq m_{1}, \nu_{1, i}^{(1)}=0} \nu_{1, i}^{(k)}+k \sum_{i=1}^{m_{k}} \nu_{k, i}^{(k)} .
$$


Hence by (16)-(19),

$$
\begin{aligned}
& \frac{1}{k}\left[(p+1)^{k}-(p+1)\right] \\
= & n_{k}+\frac{1}{k} \sum_{1 \leq i \leq m_{1}, \nu_{1, i}^{(1)}=0} \nu_{1, i}^{(k)}+\sum_{i=1}^{m_{k}} \nu_{k, i}^{(k)} \\
= & \left(n_{1}-m_{1}\right)+\left(n_{k}-m_{k}\right)-\left(n_{1}+\sum_{i=1}^{m_{1}} \nu_{1, i}^{(1)}\right)+m_{1}+m_{k} \\
& +\sum_{i=1}^{m_{1}} \nu_{1, i}^{(1)}+\frac{1}{k} \sum_{1 \leq i \leq m_{1}, \nu_{1, i}^{(1)}=0} \nu_{1, i}^{(k)}+\sum_{i=1}^{m_{k}} \nu_{k, i}^{(k)} \\
\leq & \left(n_{1}-m_{1}\right)+\left(n_{k}-m_{k}\right)-q+2\left(N_{c}-n_{\infty}\right)-\left(\sum_{i \in I} 1+\sum_{i \in J} 1\right) \\
\leq & \left(n_{1}-m_{1}\right)+\left(n_{k}-m_{k}\right)-q+2\left(N_{c}-n_{\infty}\right) .
\end{aligned}
$$

Lemma 15 (15, Theorem 4]). Let $P$ be a polynomial of degree $\geq 2$. Then for any integer $k \geq 2, P^{k}$ has at least one repelling fixed point in $\mathbb{C}$.

Remark 5. By Lemma 15 , for $k=2$ or 3 , every polynomial $P$ of degree $\geq 2$ either has at least one repelling fixed point in $\mathbb{C}$ or at least one repelling periodic cycle of period $k$ in $\mathbb{C}$. Indeed, this claim holds for all $k \geq 2$; see 9 .

We now give the proofs of Lemmas $4-7$ as follows.

Proof of Lemma 4. Suppose that the lemma does not hold, that is, $R$ has neither repelling fixed points in $\mathbb{C}$ nor repelling periodic cycles of period 4 in $\mathbb{C}$. We consider two cases.

Case 1. $\infty$ is a repelling fixed point of $R$. Then by assumption, $n_{1}=m_{1}, n_{4}=m_{4}$, and $R$ has the form (5) with $q=p+1=d$ and $0<|c+1|<1$.

Thus, by Lemma 14,

$$
\begin{aligned}
p+1 & =m_{1}+\sum_{i=1}^{m_{1}} \nu_{1, i}^{(1)}, \\
(p+1)^{2} & =m_{1}+\sum_{i=1}^{m_{1}} \nu_{1, i}^{(2)}+2 n_{2}+2 \sum_{i=1}^{m_{2}} \nu_{2, i}^{(2)}, \\
(p+1)^{4} & =m_{1}+\sum_{i=1}^{m_{1}} \nu_{1, i}^{(4)}+2 n_{2}+2 \sum_{i=1}^{m_{2}} \nu_{2, i}^{(4)}+4 m_{4}+4 \sum_{i=1}^{m_{4}} \nu_{4, i}^{(4)} .
\end{aligned}
$$

By (24) and (25),

$$
\sum_{i=1}^{m_{1}} \nu_{1, i}^{(2)}+2 \sum_{i=1}^{m_{2}} \nu_{2, i}^{(2)}+(p+1)^{4}-(p+1)^{2}=4 m_{4}+\sum_{i=1}^{m_{1}} \nu_{1, i}^{(4)}+2 \sum_{i=1}^{m_{2}} \nu_{2, i}^{(4)}+4 \sum_{i=1}^{m_{4}} \nu_{4, i}^{(4)}
$$

and by Lemma 12 ,

$$
m_{1}+m_{2}+m_{4} \leq 2 p
$$


By (10),

$$
\sum_{i=1}^{m_{1}} \nu_{1, i}^{(4)}+2 \sum_{i=1}^{m_{2}} \nu_{2, i}^{(4)}+4 \sum_{i=1}^{m_{4}} \nu_{4, i}^{(4)} \leq 8 p
$$

Thus, by (26)-(28),

$$
4\left(m_{1}+m_{2}\right)+\sum_{i=1}^{m_{1}} \nu_{1, i}^{(2)}+2 \sum_{i=1}^{m_{2}} \nu_{2, i}^{(2)}+(p+1)^{4}-(p+1)^{2} \leq 16 p .
$$

By (23) and the fact that $\nu_{1, i}^{(2)} \geq \nu_{1, i}^{(1)}\left(1 \leq i \leq m_{1}\right)$, we have

$$
m_{1}+\sum_{i=1}^{m_{1}} \nu_{1, i}^{(2)} \geq m_{1}+\sum_{i=1}^{m_{1}} \nu_{1, i}^{(1)}=p+1
$$

By (23), $m_{1} \geq 1$. Thus by (29), (30) and the facts that $m_{2} \geq 0$ and $\nu_{2, i}^{(2)} \geq 0$,

$$
(p+1)^{4}-(p+1)^{2}-15(p+1)+19 \leq 0 .
$$

This is impossible.

Case 2. $\infty$ is not a repelling fixed point of $R$. Then $R$ has no repelling fixed point and by Lemma $8, R$ has a fixed point $z_{0} \in \overline{\mathbb{C}}$ with multiplier 1 . Let $T$ be a linear transformation such that $T\left(z_{0}\right)=\infty$ and $T(\infty)=z_{0}$. Then the rational function $\widehat{R}=T \circ R \circ T^{-1}$ has a fixed point $\infty$ with multiplier 1 , has no repelling fixed points, and has at most one repelling periodic cycle of period 4 contained in $\mathbb{C}$, since $R$ has no repelling periodic cycles of period 4 contained in $\mathbb{C}$.

Thus we may assume that $\infty$ is a fixed point of $R$ with multiplier 1 , so that $R$ has the form (5) with $q \leq p$ and $d=p+1$, and by assumption, $n_{1}-m_{1}=0$, $0 \leq n_{4}-m_{4} \leq 1$.

Thus by Lemma 14,

$$
\begin{gathered}
q=m_{1}+\sum_{i=1}^{m_{1}} \nu_{1, i}^{(1)} \\
q+(p+1)^{2}-(p+1)=m_{1}+\sum_{i=1}^{m_{1}} \nu_{1, i}^{(2)}+2 n_{2}+2 \sum_{i=1}^{m_{2}} \nu_{2, i}^{(2)}, \\
q+(p+1)^{4}-(p+1)=m_{1}+\sum_{i=1}^{m_{1}} \nu_{1, i}^{(4)}+2 n_{2}+2 \sum_{i=1}^{m_{2}} \nu_{2, i}^{(4)}+4 n_{4}+4 \sum_{i=1}^{m_{4}} \nu_{4, i}^{(4)} \\
\leq m_{1}+\sum_{i=1}^{m_{1}} \nu_{1, i}^{(4)}+2 n_{2}+2 \sum_{i=1}^{m_{2}} \nu_{2, i}^{(4)}+4\left(m_{4}+1\right)+4 \sum_{i=1}^{m_{4}} \nu_{4, i}^{(4)} .
\end{gathered}
$$

By (33) and (34),

$$
\sum_{i=1}^{m_{1}} \nu_{1, i}^{(2)}+2 \sum_{i=1}^{m_{2}} \nu_{2, i}^{(2)}+(p+1)^{4}-(p+1)^{2} \leq 4\left(m_{4}+1\right)+\sum_{i=1}^{m_{1}} \nu_{1, i}^{(4)}+2 \sum_{i=1}^{m_{2}} \nu_{2, i}^{(4)}+4 \sum_{i=1}^{m_{4}} \nu_{4, i}^{(4)} .
$$


By Lemma 12, $m_{1}+m_{2}+m_{4} \leq 2 p-1$, since $\infty$ is a fixed point with multiplier 1. By Remark 1 of Lemma $13, N_{c}^{\prime} \leq 2 p-(p-q+1)=p+q-1$. Thus, by (10) and (35),

$$
4\left(m_{1}+m_{2}\right)+\sum_{i=1}^{m_{1}} \nu_{1, i}^{(2)}+2 \sum_{i=1}^{m_{2}} \nu_{2, i}^{(2)}+(p+1)^{4}-(p+1)^{2} \leq 8 p+4(p+q-1) .
$$

It follows from (36) and $q \leq p$ that $p=1, q=1$ and $m_{1}=0$. However, this contradicts (32). Lemma 4 is proved.

Proof of Lemma 5. Suppose that the lemma does not hold, that is, $R$ has neither repelling fixed points in $\mathbb{C}$ nor repelling periodic cycles of period 3 in $\mathbb{C}$. Next we consider five cases.

Case 1. $\infty$ is a repelling fixed point of $R$. Then by assumption, $n_{1}-m_{1}=n_{3}-m_{3}=$ 0 and $R$ has the form (5) with $q=p+1=d$ and $0<|c+1|<1$.

Thus, by Lemma 14,

$$
\begin{aligned}
p+1 & =m_{1}+\sum_{i=1}^{m_{1}} \nu_{1, i}^{(1)}, \\
(p+1)^{3} & =m_{1}+\sum_{i=1}^{m_{1}} \nu_{1, i}^{(3)}+3 m_{3}+3 \sum_{i=1}^{m_{3}} \nu_{3, i}^{(3)} .
\end{aligned}
$$

By Lemmas 9-11 and (16),

$$
\begin{aligned}
& m_{1}+m_{3} \\
\leq & \sum_{i \in I} 1+\sum_{i \in J} 1+\sum_{i=1}^{m_{1}} \nu_{1, i}^{(1)}+\frac{1}{3} \sum_{1 \leq i \leq m_{1}, \nu_{1, i}^{(1)}=0} \nu_{1, i}^{(3)}+\sum_{i=1}^{m_{3}} \nu_{3, i}^{(3)} \\
\leq & 2 p .
\end{aligned}
$$

Thus, by (37)-(39),

$$
\begin{aligned}
& \frac{1}{3}\left[(p+1)^{3}-(p+1)\right] \\
= & m_{3}+m_{1}-\left(m_{1}+\sum_{i=1}^{m_{1}} \nu_{1, i}^{(1)}\right)+\sum_{i=1}^{m_{1}} \nu_{1, i}^{(1)}+\frac{1}{3} \sum_{1 \leq i \leq m_{1}, \nu_{1, i}^{(1)}=0} \nu_{1, i}^{(3)}+\sum_{i=1}^{m_{3}} \nu_{3, i}^{(3)} \\
\leq & 4 p-(p+1)=3 p-1 .
\end{aligned}
$$

It follows that $p=1, m_{1}+m_{3}=2$. By (37), $1 \leq m_{1} \leq p+1=2$, so that either $m_{1}=m_{3}=1$ or $m_{1}=2$ and $m_{3}=0$.

If $m_{1}=m_{3}=1$, then by $(40), \nu_{1,1}^{(1)}=\nu_{1,1}^{(3)}=1$ and $\nu_{3,1}^{(3)}=1$. Thus

$$
\begin{aligned}
& P(z)=z-a, Q(z)=(z-b)^{2}, \\
& Q_{3}(z)=(z-b)^{2}\left[\left(z-z_{1}\right)\left(z-R\left(z_{1}\right)\right)\left(z-R^{2}\left(z_{1}\right)\right)\right]^{2},
\end{aligned}
$$

where $a, b$ are distinct constants and $\left\{z_{1}, R\left(z_{1}\right), R^{2}\left(z_{1}\right)\right\} \subset \mathbb{C}$. By a suitable conjugation $z \rightarrow \alpha z+\beta$, we may assume $a=1$ and $b=0$. Thus

$$
R(z)=z+c \frac{z^{2}}{z-1} \text {. }
$$


A computation shows that $Q_{3}(z)=z^{2} H(z)$, where

$$
\begin{gathered}
H(z)=z^{6}-\frac{4 c+6}{(c+1)^{2}} z^{5}+\frac{8 c+15}{(c+1)^{3}} z^{4}-\frac{10 c^{3}+52 c^{2}+96 c+60}{\left(c^{2}+3 c+3\right)(c+1)^{4}} z^{3} \\
+\frac{9 c^{2}+39 c+45}{\left(c^{2}+3 c+3\right)(c+1)^{4}} z^{2}-\frac{6 c+18}{\left(c^{2}+3 c+3\right)(c+1)^{4}} z+\frac{3}{\left(c^{2}+3 c+3\right)(c+1)^{4}}
\end{gathered}
$$

It follows from $Q_{3}(z)=z^{2} H(z)$ and (41) with $b=0$ that $H$ is a square of a cubic polynomial, say

$$
H(z)=\left(z^{3}+\alpha z^{2}+\beta z+\gamma\right)^{2}
$$

$$
=z^{6}+2 \alpha z^{5}+\left(\alpha^{2}+2 \beta\right) z^{4}+(2 \gamma+2 \alpha \beta) z^{3}+\left(2 \alpha \gamma+\beta^{2}\right) z^{2}+2 \beta \gamma z+\gamma^{2},
$$

where $\alpha, \beta, \gamma$ are constants. Equating coefficients in (42) and (43) and solving the equations obtained yields that $c=-3 / 2$. Thus in this case, $R$ is affinely conjugate to the first function stated in Lemma 5 .

If $m_{1}=2$ and $m_{3}=0$, then by $(40), \nu_{1,1}^{(1)}=\nu_{1,2}^{(1)}=0$ and $\nu_{1,1}^{(3)}=\nu_{1,2}^{(3)}=3$. Thus

$$
P(z)=z-a, Q(z)=\left(z-b_{1}\right)\left(z-b_{2}\right), Q_{3}(z)=\left(z-b_{1}\right)^{4}\left(z-b_{2}\right)^{4} .
$$

By a suitable conjugation $z \rightarrow \alpha z+\beta$, we may assume $b_{1}=1$ and $b_{2}=0$. Thus

$$
R(z)=z+c \frac{z(z-1)}{z-a},
$$

with $a \neq 0,1$. A computation shows that $Q_{3}(z)=z(z-1) H(z)$, where

$$
\begin{gathered}
H(z)=z^{6}-\frac{A_{5}}{(c+1)^{2}} z^{5}+\frac{A_{4}}{(c+1)^{3}} z^{4}-\frac{A_{3}}{\left(c^{2}+3 c+3\right)(c+1)^{4}} z^{3} \\
+\frac{A_{2}}{\left(c^{2}+3 c+3\right)(c+1)^{4}} z^{2}-\frac{A_{1}}{\left(c^{2}+3 c+3\right)(c+1)^{4}} z+\frac{A_{0}}{\left(c^{2}+3 c+3\right)(c+1)^{4}},
\end{gathered}
$$

with

$$
\begin{aligned}
A_{5}= & (4 c+6) a+3 c^{2}+4 c, \\
A_{4}= & (8 c+15) a^{2}+\left(10 c^{2}+17 c\right) a+3 c^{3}+5 c^{2}, \\
A_{3}= & \left(10 c^{3}+52 c^{2}+96 c+60\right) a^{3}+\left(16 c^{4}+79 c^{3}+138 c^{2}+84 c\right) a^{2} \\
& +\left(8 c^{5}+38 c^{4}+65 c^{3}+40 c^{2}\right) a+c^{6}+5 c^{5}+9 c^{4}+6 c^{3}, \\
A_{2}= & \left(9 c^{2}+39 c+45\right) a^{4}+\left(15 c^{3}+60 c^{2}+66 c\right) a^{3} \\
& +\left(9 c^{4}+34 c^{3}+36 c^{2}\right) a^{2}+\left(2 c^{5}+7 c^{4}+7 c^{3}\right) a, \\
A_{1}= & (6 c+18) a^{5}+\left(9 c^{2}+24 c\right) a^{4}+\left(5 c^{3}+12 c^{2}\right) a^{3}+\left(c^{4}+2 c^{3}\right) a^{2}, \\
A_{0}= & 3 a^{6}+3 c a^{5}+c^{2} a^{4} .
\end{aligned}
$$

From $Q_{3}(z)=z(z-1) H(z)$ and (44) with $b_{1}=1$ and $b_{2}=0$, we obtain $H=$ $z^{3}(z-1)^{3}$. It follows from (45) that $a=1 / 2$ and $c=(-3 \pm \sqrt{3} i) / 4$. In this case, $R$ is affinely conjugate to the second function stated in Lemma 5 .

Case 2. $\infty$ is a fixed point of $R$ with multiplier 1 , so that $R$ has the form (5) with $q \leq p$ and $d=p+1$, and by assumption, $n_{1}=m_{1}, n_{3}=m_{3}$. 
Thus, by Lemma 14,

$$
\begin{gathered}
q=m_{1}+\sum_{i=1}^{m_{1}} \nu_{1, i}^{(1)}, \\
q+(p+1)^{3}-(p+1)=m_{1}+\sum_{i=1}^{m_{1}} \nu_{1, i}^{(3)}+3 m_{3}+3 \sum_{i=1}^{m_{3}} \nu_{3, i}^{(3)} .
\end{gathered}
$$

Thus, as above, we have

$$
\begin{aligned}
& \frac{1}{3}\left[(p+1)^{3}-(p+1)\right] \\
= & m_{3}+m_{1}-\left(m_{1}+\sum_{i=1}^{m_{1}} \nu_{1, i}^{(1)}\right)+\sum_{i=1}^{m_{1}} \nu_{1, i}^{(1)}+\frac{1}{3} \sum_{1 \leq i \leq m_{1}, \nu_{1, i}^{(1)}=0} \nu_{1, i}^{(3)}+\sum_{i=1}^{m_{3}} \nu_{3, i}^{(3)} \\
\leq & 2\left(2 p-n_{\infty}\right)-q \\
\leq & 2 p+q-2 \leq 3 p-2 .
\end{aligned}
$$

This is impossible.

Case 3. $\infty$ is a fixed point of $R$ but not a weakly repelling fixed point of $R$. Then by Lemma 8 and the assumption that $R$ has no repelling fixed points in $\mathbb{C}, R$ has a fixed point $z_{0} \in \mathbb{C}$ with multiplier 1 . Let

$$
\phi(z)=z_{0}+\frac{1}{z-z_{0}} .
$$

Define

$$
\widetilde{R}(z)=\phi^{-1} \circ R \circ \phi(z) .
$$

Then $\infty$ is a fixed point of $\widetilde{R}$ with multiplier 1 , so that $\widetilde{R}$ has the form (5) with $q \leq p$ and $d=p+1$, and by assumption, $n_{1}=m_{1}, n_{3}=m_{3}$, where $n_{j}\left(m_{j}\right)$ denotes the number of (non-repelling) periodic cycles of period $j$ of $\widetilde{R}$ in $\mathbb{C}$.

By Case 2, this is impossible.

Case $4 . \infty$ is a periodic point of $R$ of period 3. Then by Lemma 8 and the assumption that $R$ has no repelling fixed points in $\mathbb{C}, R$ has a fixed point $z_{0} \in \mathbb{C}$ with multiplier 1. Let $\phi$ be defined in (48) and $\widetilde{R}$ be defined in (49). Then $\infty$ is a fixed point of $\widetilde{R}$ with multiplier 1 , so that $\widetilde{R}$ has the form (5) with $q \leq p$ and $d=p+1$, and by assumption, $n_{1}=m_{1}, n_{3} \leq m_{3}+1$, where $n_{j}\left(m_{j}\right)$ denotes the number of (non-repelling) periodic cycles of period $j$ of $\widetilde{R}$ in $\mathbb{C}$. By Case 2, $n_{3}=m_{3}+1$. Therefore, $z_{0}$ is a repelling periodic point of $\widetilde{R}$ of period 3 .

Thus, by applying Lemma 14 to $\widetilde{R}$, we have

$$
\begin{aligned}
q & =m_{1}+\sum_{i=1}^{m_{1}} \nu_{1, i}^{(1)}, \\
q+(p+1)^{3}-(p+1) & =m_{1}+\sum_{i=1}^{m_{1}} \nu_{1, i}^{(3)}+3\left(m_{3}+1\right)+3 \sum_{i=1}^{m_{3}} \nu_{3, i}^{(3)} .
\end{aligned}
$$


Hence by (50), (51) and Remark 4,

$$
\begin{aligned}
& \frac{1}{3}\left[(p+1)^{3}-(p+1)\right] \\
= & 1+m_{3}+m_{1}-\left(m_{1}+\sum_{i=1}^{m_{1}} \nu_{1, i}^{(1)}\right)+\sum_{i=1}^{m_{1}} \nu_{1, i}^{(1)}+\frac{1}{3} \sum_{1 \leq i \leq m_{1}, \nu_{1, i}^{(1)}=0} \nu_{1, i}^{(3)}+\sum_{i=1}^{m_{3}} \nu_{3, i}^{(3)} \\
\leq & 1+2\left(2 p-n_{\infty}\right)-q-\sum_{i \in I} 1-\sum_{i \in J} 1 \\
\leq & 2 p+q-1 \leq 3 p-1 .
\end{aligned}
$$

It follows that $p=1, q=1, m_{1}=1, m_{3}=0, \nu_{1,1}^{(1)}=0$ and $\nu_{1,1}^{(3)}=3$. Thus $\widetilde{R}$ has the form

$$
\widetilde{R}(z)=z+c \frac{z+b}{z+a},
$$

where $a, b$ are distinct constants, and

(54) $\widetilde{R}^{3}(z)=z+c_{3} \frac{\widetilde{Q}_{3}(z)}{\widetilde{P}_{3}(z)}$ with $\widetilde{Q}_{3}(z)=(z+b)^{4}\left(z-z_{1}\right)\left(z-R\left(z_{1}\right)\right)\left(z-R^{2}\left(z_{1}\right)\right)$,

where $\left\{z_{1}, R\left(z_{1}\right), R^{2}\left(z_{1}\right)\right\} \subset \mathbb{C}$. By a suitable conjugation $z \rightarrow \tau z+\omega$, we may assume $a=1$ and $b=0$. After some computation, we have

$$
\begin{aligned}
& \widetilde{Q}_{3}(z)=z\left[z^{6}+(4 c+6) z^{5}+\left(5 c^{2}+17 c+15\right) z^{4}+\left(2 c^{3}+\frac{40}{3} c^{2}+28 c+20\right) z^{3}\right. \\
& \left.+\left(\frac{7}{3} c^{3}+12 c^{2}+22 c+15\right) z^{2}+\left(\frac{2}{3} c^{3}+4 c^{2}+8 c+6\right) z+\frac{1}{3} c^{2}+c+1\right] .
\end{aligned}
$$

This, with (54) $(b=0)$, shows that $c^{2}+3 c+3=0$, so that

$$
c=\frac{-3 \pm \sqrt{3} i}{2} \text {. }
$$

Computation then yields

$$
\widetilde{R}^{3}(z)=z+\frac{(-18 \pm 6 \sqrt{3} i) z^{4}\left[z^{3} \pm 2 \sqrt{3} i z^{2}+(-3 \pm \sqrt{3} i) z-2\right]}{\left[2 z^{4}+(-1 \pm 3 \sqrt{3} i) z^{3}+2 z+2\right]\left[2 z^{2}+(1 \pm \sqrt{3} i) z+2\right](z+1)} .
$$

Since $z_{0}$ is a periodic point of $\widetilde{R}$ of period 3 ,

$$
z_{0}^{3} \pm 2 \sqrt{3} i z_{0}^{2}+(-3 \pm \sqrt{3} i) z_{0}-2=0 .
$$

Thus by (48), (49) and (53) with $a=1$ and $b=0$,

$$
R(z)=\phi^{-1} \circ \widetilde{R} \circ \phi(z)=z-\frac{c\left(z-z_{0}\right)^{2}\left[z_{0}\left(z-z_{0}\right)+1\right]}{c z_{0}\left(z-z_{0}\right)^{2}+\left(c+z_{0}+1\right)\left(z-z_{0}\right)+1},
$$

where the constants $c, z_{0}$ satisfy $c^{2}+3 c+3=0$ and $z_{0}^{3}+(4 c+6) z_{0}^{2}+2 c z_{0}-2=0$. Thus in this case, $R$ is affinely conjugate to the third function stated in Lemma 5 .

Case $5 . \infty$ is not a fixed point of $R^{3}$. Then by the assumption that $R$ has no repelling fixed points in $\mathbb{C}$ and Lemma $8, R$ has a fixed point $z_{0} \in \mathbb{C}$ with multiplier 1. Then $\infty$ is a fixed point of the function $\widetilde{R}$ defined in (49) with multiplier 1 , and $n_{1}=m_{1}, n_{3}=m_{3}$. By Case 2, this is impossible. 
Lemma 5 is proved.

Proof of Lemma 6. By Lemma 15, we may assume that $R$ is not a polynomial. Thus $R$ has least one pole in $\mathbb{C}$. By assumption, the multiplicity of this pole is at least 3 , and hence $d=\operatorname{deg}(R) \geq 3$.

Now suppose that the lemma does not hold, that is, $R$ has neither repelling fixed points in $\mathbb{C}$ nor repelling periodic cycles of period 2 in $\mathbb{C}$. We consider two cases.

Case $1 . \infty$ is a fixed point of $R$. We claim that $d \geq 4$ and that

$$
N_{c} \leq \frac{5}{3}(d-1) \text { and } N_{c}^{\prime}+N_{c}^{\prime \prime} \leq \frac{4}{3}(d-1)
$$

In fact, we have

$$
R(z)=\frac{U(z)}{V(z)}
$$

with $d=\operatorname{deg}(R)=u=\operatorname{deg}(U) \geq \operatorname{deg}(V)+1=v+1$ and

$$
V(z)=\prod_{j=1}^{t}\left(z-z_{j}\right)^{s_{j}}
$$

with $s_{j} \geq 3$, so that $v=\sum_{j=1}^{t} s_{j} \geq 3 t \geq 3$. Thus $d \geq 4$. Computation shows that

$$
R^{\prime}(z)=\frac{U^{\prime} \prod_{j=1}^{t}\left(z-z_{j}\right)-U \sum_{j=1}^{t} s_{j} \prod_{i \neq j}\left(z-z_{i}\right)}{\prod_{j=1}^{t}\left(z-z_{j}\right)^{s_{j}+1}} .
$$

Thus $N_{c}^{\prime}+N_{c}^{\prime \prime} \leq u-1+t \leq \frac{4}{3}(d-1)$ and $N_{c} \leq u-1+t+t \leq \frac{5}{3}(d-1)$. This proves $(59)$.

Case 1.1. $\infty$ is a repelling fixed point of $R$. Then by assumption, $n_{1}-m_{1}=$ $n_{2}-m_{2}=0$ and $R$ has the form (5) with $q=p+1=d$ and $0<|c+1|<1$. By $(59)$

$$
N_{c} \leq \frac{5}{3} p
$$

By Lemma 14,

$$
\begin{aligned}
p+1 & =m_{1}+\sum_{i=1}^{m_{1}} \nu_{1, i}^{(1)}, \\
(p+1)^{2} & =m_{1}+\sum_{i=1}^{m_{1}} \nu_{1, i}^{(2)}+2 m_{2}+2 \sum_{i=1}^{m_{2}} \nu_{2, i}^{(2)} .
\end{aligned}
$$

By Lemmas 9-11, (16) and (60),

$$
\begin{aligned}
& m_{1}+m_{2} \\
\leq & \sum_{i \in I} 1+\sum_{i \in J} 1+\sum_{i=1}^{m_{1}} \nu_{1, i}^{(1)}+\frac{1}{2} \sum_{1 \leq i \leq m_{1}, \nu_{1, i}^{(1)}=0} \nu_{1, i}^{(2)}+\sum_{i=1}^{m_{2}} \nu_{2, i}^{(2)} \\
\leq & \frac{5}{3} p .
\end{aligned}
$$


Thus by (61)-(64), we get

$$
\begin{aligned}
& \frac{1}{2}\left[(p+1)^{2}-(p+1)\right] \\
= & m_{2}+m_{1}-\left(m_{1}+\sum_{i=1}^{m_{1}} \nu_{1, i}^{(1)}\right)+\sum_{i=1}^{m_{1}} \nu_{1, i}^{(1)}+\frac{1}{2} \sum_{1 \leq i \leq m_{1}, \nu_{1, i}^{(1)}=0} \nu_{1, i}^{(2)}+\sum_{i=1}^{m_{2}} \nu_{2, i}^{(2)} \\
\leq & \frac{10}{3} p-(p+1)-\left(\sum_{i \in I} 1+\sum_{i \in J} 1\right) \\
\leq & \frac{7}{3} p-1 .
\end{aligned}
$$

It follows that $p=3$ and $\sum_{i \in I} 1+\sum_{i \in J} 1=0$. Thus $d=4$, and by (63), (12) and (59),

$$
m_{1}+m_{2} \leq \sum_{i=1}^{m_{1}} \nu_{1, i}^{(1)}+\frac{1}{2} \sum_{1 \leq i \leq m_{1}, \nu_{1, i}^{(1)}=0} \nu_{1, i}^{(2)}+\sum_{i=1}^{m_{2}} \nu_{2, i}^{(2)} \leq 4 .
$$

Thus by (61), (65) and (66), we get a contradiction: $6 \leq 8-4=4$.

Case 1.2. $\infty$ is a fixed point of $R$ with multiplier 1 . Then by assumption, $n_{1}-m_{1}=$ $n_{2}-m_{2}=0$ and $R$ has the form (5) with $q \leq p, p+1=d$. We also have (60).

Thus, by Lemma 14,

$$
\begin{gathered}
q=m_{1}+\sum_{i=1}^{m_{1}} \nu_{1, i}^{(1)}, \\
q+(p+1)^{2}-(p+1)=m_{1}+\sum_{i=1}^{m_{1}} \nu_{1, i}^{(2)}+2 m_{2}+2 \sum_{i=1}^{m_{2}} \nu_{2, i}^{(2)} .
\end{gathered}
$$

By Lemmas 9-11, (16) and (60),

$$
\begin{aligned}
& m_{1}+m_{2} \\
\leq & \sum_{i \in I} 1+\sum_{i \in J} 1+\sum_{i=1}^{m_{1}} \nu_{1, i}^{(1)}+\frac{1}{2} \sum_{1 \leq i \leq m_{1}, \nu_{1, i}^{(1)}=0} \nu_{1, i}^{(2)}+\sum_{i=1}^{m_{2}} \nu_{2, i}^{(2)} \\
\leq & \frac{5}{3} p-(p-q+1)=\frac{2}{3} p+q-1 .
\end{aligned}
$$

Thus by (67)-(69), we have

$$
\begin{aligned}
& \frac{1}{2}\left[(p+1)^{2}-(p+1)\right] \\
= & m_{2}+m_{1}-\left(m_{1}+\sum_{i=1}^{m_{1}} \nu_{1, i}^{(1)}\right)+\sum_{i=1}^{m_{1}} \nu_{1, i}^{(1)}+\frac{1}{2} \sum_{1 \leq i \leq m_{1}, \nu_{1, i}^{(1)}=0} \nu_{1, i}^{(2)}+\sum_{i=1}^{m_{2}} \nu_{2, i}^{(2)} \\
\leq & 2\left(\frac{2}{3} p+q-1\right)-q-\left(\sum_{i \in I} 1+\sum_{i \in J} 1\right) \\
\text { (70) } \leq & \frac{4}{3} p+q-2 \leq \frac{7}{3} p-2 .
\end{aligned}
$$

It follows that $p=1$ so that $d=2$, a contradiction. 
Case 1.3. $\infty$ is a fixed point of $R$, but not a weakly repelling fixed point of $R$. Since $R$ has no repelling fixed point in $\mathbb{C}$, by Lemma $8, R$ has a fixed point $z_{0} \in \mathbb{C}$ with multiplier 1 . Let

$$
\phi(z)=z_{0}+\frac{1}{z-z_{0}} .
$$

Define

$$
\widetilde{R}(z)=\phi^{-1} \circ R \circ \phi(z) .
$$

Then $\infty$ is a fixed point of $\widetilde{R}$ with multiplier 1 , so that $\widetilde{R}$ has the form (5) with $q \leq p$ and $\operatorname{deg}(\widetilde{R})=p+1$, and by assumption, $n_{1}=m_{1}, n_{2}=m_{2}$, where $n_{j}\left(m_{j}\right)$ denotes the number of (non-repelling) periodic cycles of period $j$ of $\widetilde{R}$ in $\mathbb{C}$. By $(59)$

$$
N_{c} \leq \frac{5}{3} p
$$

Then, as in Case 1.2, we get (70), and hence $p=1$ so that $d=2$, a contradiction.

Case 2. $\infty$ is not a fixed point of $R$. We claim that

$$
N_{c} \leq \frac{5}{3} d-2 \text {. }
$$

In fact, since $\infty$ is not a fixed point of $R, R$ can be written as

$$
R(z)=c+\frac{U(z)}{V(z)}
$$

where $c$ is a constant, $u=\operatorname{deg}(U)<\operatorname{deg}(V)=v=d=\operatorname{deg}(R)$. Then as in Case 1, $N_{c} \leq u-1+2 t \leq \frac{5}{3} d-2$. This proves the claim.

Case 2.1. $\infty$ is a periodic point of $R$ of period 2. Again since $R$ has no repelling fixed point in $\mathbb{C}$, by Lemma $8, R$ has a fixed point $z_{0} \in \mathbb{C}$ with multiplier 1 . Let $\phi(z)$ and $\widetilde{R}$ be as in Case 1.3. Then $\infty$ is a fixed point of $\widetilde{R}$ with multiplier 1 so that $\widetilde{R}$ has the form (5) with $q \leq p$ and $d=p+1$, and by assumption, $n_{1}=m_{1}$, $n_{2} \leq m_{2}+1$, where $n_{j}\left(m_{j}\right)$ denotes the number of (non-repelling) periodic cycles of period $j$ of $\widetilde{R}$ in $\mathbb{C}$, since $z_{0}$ may be a repelling periodic point of $\widetilde{R}$ of period 2 . By (74),

$$
N_{c} \leq \frac{1}{3}(5 p-1)
$$

By Lemma 14,

$$
\begin{aligned}
q & =m_{1}+\sum_{i=1}^{m_{1}} \nu_{1, i}^{(1)}, \\
q+(p+1)^{2}-(p+1) & =m_{1}+\sum_{i=1}^{m_{1}} \nu_{1, i}^{(2)}+2 n_{2}+2 \sum_{i=1}^{m_{2}} \nu_{2, i}^{(2)} \\
& \leq 2+m_{1}+\sum_{i=1}^{m_{1}} \nu_{1, i}^{(2)}+2 m_{2}+2 \sum_{i=1}^{m_{2}} \nu_{2, i}^{(2)} .
\end{aligned}
$$


By Lemmas 9-11, (16) and (75),

$$
\begin{aligned}
& m_{1}+m_{2} \\
\leq & \sum_{i \in I} 1+\sum_{i \in J} 1+\sum_{i=1}^{m_{1}} \nu_{1, i}^{(1)}+\frac{1}{2} \sum_{1 \leq i \leq m_{1}, \nu_{1, i}^{(1)}=0} \nu_{1, i}^{(2)}+\sum_{i=1}^{m_{2}} \nu_{2, i}^{(2)} \\
\leq & N_{c}-(p-q+1) \leq \frac{1}{3}(2 p-4)+q .
\end{aligned}
$$

Thus, by (76)-(78), we have

$$
\begin{aligned}
& \frac{1}{2}\left[(p+1)^{2}-(p+1)\right] \\
\leq & 1+m_{1}+m_{2}-\left(m_{1}+\sum_{i=1}^{m_{1}} \nu_{1, i}^{(1)}\right)+\sum_{i=1}^{m_{1}} \nu_{1, i}^{(1)}+\frac{1}{2} \sum_{1 \leq i \leq m_{1}, \nu_{1, i}^{(1)}=0} \nu_{1, i}^{(2)}+\sum_{i=1}^{m_{2}} \nu_{2, i}^{(2)} \\
\leq & 1+2\left[\frac{1}{3}(2 p-4)+q\right]-q-\left(\sum_{i \in I} 1+\sum_{i \in J} 1\right) \\
\leq & \frac{7 p-5}{3}
\end{aligned}
$$

This is impossible.

Case 2.2. $\infty$ is not a fixed point of $R^{2}$. Then by Lemma 8 and the assumption that $R$ has no repelling fixed point in $\mathbb{C}, R$ has a fixed point $z_{0} \in \mathbb{C}$ with multiplier 1 . Thus $\infty$ is a fixed point of the function $\widetilde{R}$ defined in (60) with multiplier 1 , and $n_{1}=m_{1}, n_{2}=m_{2}$. In a similar way, this case cannot occur.

Lemma 6 is proved.

Proof of Lemma 7. Suppose that the lemma does not hold. Then $R$ has no weakly repelling fixed point in $\mathbb{C}$, and thus by Lemma $8, \infty$ must be a weakly repelling fixed point of $R$. We consider two cases.

Case 1. $\infty$ is a repelling fixed point of $R$. Then by assumption, $n_{1}-m_{1}=n_{2}-m_{2}=$ 0 , and $R$ has the form (5) with $q=p+1=d$ and $0<|c+1|<1$.

Thus by Lemma 14,

$$
\begin{aligned}
p+1 & =m_{1}+\sum_{i=1}^{m_{1}} \nu_{1, i}^{(1)}, \\
(p+1)^{2} & =m_{1}+\sum_{i=1}^{m_{1}} \nu_{1, i}^{(2)}+2 m_{2}+2 \sum_{i=1}^{m_{2}} \nu_{2, i}^{(2)} .
\end{aligned}
$$

By assumption, $\nu_{1, i}^{(1)}=\nu_{1, i}^{(2)}=0$. Then $m_{1}=\sum_{i \in I} 1=p+1$, and by (16) with $N_{c} \leq 2 p$

$$
m_{2} \leq \sum_{i \in J} 1+\sum_{i=1}^{m_{2}} \nu_{2, i}^{(2)} \leq p-1
$$


Thus by (80) and (81),

$$
(p+1)^{2}=p+1+2 m_{2}+2 \sum_{i=1}^{m_{2}} \nu_{2, i}^{(2)} \leq 5 p-3,
$$

which is impossible.

Case $2 . \infty$ is a fixed point of $R$ with multiplier 1 . Then by assumption, $n_{1}-m_{1}=$ $n_{2}-m_{2}=0$, and $R$ has the form (5) with $q \leq p, p+1=d$.

Thus, by Lemma 14,

$$
\begin{gathered}
q=m_{1}+\sum_{i=1}^{m_{1}} \nu_{1, i}^{(1)}, \\
q+(p+1)^{2}-(p+1)=m_{1}+\sum_{i=1}^{m_{1}} \nu_{1, i}^{(2)}+2 m_{2}+2 \sum_{i=1}^{m_{2}} \nu_{2, i}^{(2)} .
\end{gathered}
$$

By assumption, $\nu_{1, i}^{(1)}=\nu_{1, i}^{(2)}=0$. Then $m_{1}=\sum_{i \in I} 1=q$, and by (16),

$$
m_{2} \leq \sum_{i \in J} 1+\sum_{i=1}^{m_{2}} \nu_{2, i}^{(2)} \leq p-1
$$

Thus, by (83) and (84),

$$
(p+1)^{2}-(p+1)=2 m_{2}+2 \sum_{i=1}^{m_{2}} \nu_{2, i}^{(2)} \leq 4 p-4,
$$

which is impossible.

This completes the proof.

We also require the following result of Pang and Zalcman.

Lemma 16 ([19, Lemma 2]; cf. [18, 25, 26]). Let $\mathcal{F}$ be a family of meromorphic functions in a domain $D$, all of whose zeros have multiplicity at least $k$, and suppose that there exists $A \geq 1$ such that $\left|f^{(k)}(z)\right| \leq A$ whenever $f(z)=0$ and $f \in \mathcal{F}$. Then if $\mathcal{F}$ is not normal at $z_{0}$, there exist, for each $0 \leq \alpha \leq k$,

a) points $z_{n} \in D, z_{n} \rightarrow z_{0}$,

b) functions $f_{n} \in \mathcal{F}$, and

c) positive numbers $\rho_{n} \rightarrow 0$

such that $\rho_{n}^{-\alpha} f_{n}\left(z_{n}+\rho_{n} \zeta\right)=g_{n}(\zeta) \rightarrow g(\zeta)$ locally uniformly with respect to the spherical metric, where $g$ is a non-constant meromorphic function in $\mathbb{C}$, all of whose zeros have multiplicity at least $k$, such that

$$
\frac{\left|g^{\prime}(\zeta)\right|}{1+|g(\zeta)|^{2}} \leq \frac{\left|g^{\prime}(0)\right|}{1+|g(0)|^{2}}=k A+1
$$

We shall use the special case $\alpha=k=1$ of Lemma 16 .

Lemma 17. Let $k \geq 2$ be an integer and $\mathcal{F}$ a family of functions meromorphic in a domain $D$ such that each $f \in \mathcal{F}$ has neither repelling fixed points in $D$ nor repelling periodic points of period $k$ in $D$. If $\mathcal{F}$ is not normal at some point $z_{0} \in D$, then there exist points $z_{n} \in D$ with $z_{n} \rightarrow z_{0}$, functions $f_{n} \in \mathcal{F}$ and positive numbers $\rho_{n} \rightarrow 0$ such that

$$
H_{n}(\zeta)=\frac{f_{n}\left(z_{n}+\rho_{n} \zeta\right)-z_{n}}{\rho_{n}} \rightarrow H(\zeta)
$$


locally uniformly with respect to the spherical metric, where $H$ is a non-constant rational function, not of the form $\zeta+c$ with constant $c \in \mathbb{C}$, such that $H$ has neither repelling fixed points in $\mathbb{C}$ nor repelling periodic points of period $k$ in $\mathbb{C}$, and

$$
\frac{\left|H^{\prime}(\zeta)-1\right|}{1+|H(\zeta)-\zeta|^{2}} \leq \frac{\left|H^{\prime}(0)-1\right|}{1+|H(0)|^{2}}=3 \text {. }
$$

Furthermore, if each $f \in \mathcal{F}$ has no fixed point in $D$ with multiplier 1 , then $\infty$ is a weakly repelling fixed point of $H$ unless $\operatorname{deg}(H)=1$.

Proof. Set

$$
\mathcal{G}=\{g=f-i d: f \in \mathcal{F}\},
$$

where $i d$ denotes the identity function. Then for every $g \in \mathcal{G},\left|g^{\prime}(z)\right| \leq 2$ whenever $g(z)=0$, since each $f \in \mathcal{F}$ has no repelling fixed points in $D$.

Obviously, $\mathcal{F}$ is normal in $D$ if and only if $\mathcal{G}$ is normal in $D$. Thus $\mathcal{G}$ is not normal at $z_{0} \in D$. Hence by Lemma 16 , we can find points $z_{n} \rightarrow z_{0}$, positive numbers $\rho_{n} \rightarrow 0$ and functions $g_{n}=f_{n}-i d \in \mathcal{G}$ such that

$$
G_{n}(\zeta)=\frac{g_{n}\left(z_{n}+\rho_{n} \zeta\right)}{\rho_{n}} \rightarrow G(\zeta)
$$

locally uniformly with respect to the spherical metric on $\mathbb{C}$, where $G$ is a nonconstant meromorphic function on $\mathbb{C}$ such that

$$
\frac{\left|G^{\prime}(\zeta)\right|}{1+|G(\zeta)|^{2}} \leq \frac{\left|G^{\prime}(0)\right|}{1+|G(0)|^{2}}=3
$$

Set

$$
\begin{aligned}
M_{n}(\zeta) & =z_{n}+\rho_{n} \zeta, \\
H_{n}(\zeta) & =G_{n}(\zeta)+\zeta .
\end{aligned}
$$

Then by (93) and (95)-(96),

$$
H_{n}(\zeta)=\frac{f_{n}\left(M_{n}(\zeta)\right)-z_{n}}{\rho_{n}} .
$$

This with (95) yields $M_{n}\left(H_{n}(\zeta)\right)=z_{n}+\rho_{n} H_{n}(\zeta)=f_{n}\left(M_{n}(\zeta)\right)$. Hence we get

$$
H_{n}^{2}(\zeta)=H_{n}\left(H_{n}(\zeta)\right)=\frac{f_{n}\left(M_{n}\left(H_{n}(\zeta)\right)\right)-z_{n}}{\rho_{n}}=\frac{f_{n}^{2}\left(M_{n}(\zeta)\right)-z_{n}}{\rho_{n}} .
$$

By mathematical induction,

$$
H_{n}^{j}(\zeta)=\frac{f_{n}^{j}\left(M_{n}(\zeta)\right)-z_{n}}{\rho_{n}}, j=1,2, \cdots,
$$

so that

$$
f_{n}^{j}\left(M_{n}(\zeta)\right)=z_{n}+\rho_{n} H_{n}^{j}(\zeta), j=1,2, \cdots
$$

and

$$
H_{n}^{j}(\zeta)-\zeta=\frac{f_{n}^{j}\left(M_{n}(\zeta)\right)-M_{n}(\zeta)}{\rho_{n}}, j=1,2, \cdots
$$

Let

$$
H(\zeta)=G(\zeta)+\zeta
$$


and

$$
A=\bigcup_{j=1}^{k+1} H^{-j}(\infty)=\bigcup_{j=1}^{k+1}\left\{\zeta \in \mathbb{C}: H^{j}(\zeta)=\infty\right\} .
$$

Then by (93), (96) and (101)-(102), for any $j \in\{1,2, \cdots, k\}$, as $n \rightarrow \infty$,

$$
H_{n}^{j}(\zeta) \rightarrow H^{j}(\zeta)
$$

locally uniformly on $\mathbb{C} \backslash A$. Note that by (94) and $(101), H(\zeta) \not \equiv \zeta+c$ for some constant $c \in \mathbb{C}$.

Claim 1. $H$ has no repelling fixed point in $\mathbb{C}$.

Let $\zeta_{0} \in \mathbb{C}$ be a fixed point of $H$. Then $H$ and $H_{n}$ for all sufficiently large $n$ are holomorphic in some neighborhood of $\zeta_{0}$. Thus, by Hurwitz's Theorem, there exist points $\zeta_{n} \rightarrow \zeta_{0}$ such that $H_{n}\left(\zeta_{n}\right)=\zeta_{n}$. By (95) and (97), we see that $f_{n}\left(z_{n}+\rho_{n} \zeta_{n}\right)=z_{n}+\rho_{n} \zeta_{n}$, i.e., $z_{n}+\rho_{n} \zeta_{n}$ is a fixed point of $f_{n}$ in $D$. Since $f_{n}$ has no repelling fixed point in $D$, we have $\left|f_{n}^{\prime}\left(z_{n}+\rho_{n} \zeta_{n}\right)\right| \leq 1$. This with (95) and (97) shows that $\left|H_{n}^{\prime}\left(\zeta_{n}\right)\right| \leq 1$. Hence

$$
\left|H^{\prime}\left(\zeta_{0}\right)\right|=\left|\lim _{n \rightarrow \infty} H_{n}^{\prime}\left(\zeta_{n}\right)\right| \leq 1 .
$$

Claim 1 is proved.

Claim 2. $H$ has no repelling periodic cycle of period $k$ in $\mathbb{C}$.

If $H^{k}(\zeta) \equiv \zeta$, then there is nothing to prove, so we may assume that $H^{k}(\zeta) \not \equiv \zeta$.

Let $\left\{\zeta_{0}, H\left(\zeta_{0}\right), \cdots, H^{k-1}\left(\zeta_{0}\right)\right\} \subset \mathbb{C}$ be a periodic cycle of period $k$ of $H$. Then for $j \in\{1,2, \cdots, k-1\}, H^{j}\left(\zeta_{0}\right) \in \mathbb{C} \backslash\left\{\zeta_{0}\right\}$ and $H^{k}\left(\zeta_{0}\right)=\zeta_{0}$. Thus there exist positive numbers $\delta$ and $\varepsilon$ such that $H^{j}(1 \leq j \leq k)$ are holomorphic on $\bar{U}=\left\{\zeta:\left|\zeta-\zeta_{0}\right| \leq \delta\right\} \subset \mathbb{C} \backslash A$, and for $j \in\{1,2, \cdots, k-1\},\left|H^{j}(\zeta)-\zeta\right| \geq \varepsilon$ on $\bar{U}$.

Thus by (103) and Hurwitz's Theorem, there exist points $\zeta_{n} \rightarrow \zeta_{0}$ such that $H_{n}^{k}\left(\zeta_{n}\right)=\zeta_{n}$, and for sufficiently large $n,\left|H_{n}^{j}\left(\zeta_{n}\right)-\zeta_{n}\right| \geq \varepsilon / 2$ for $1 \leq j \leq k-1$. Hence by $(100), f_{n}^{k}\left(M_{n}\left(\zeta_{n}\right)\right)=M_{n}\left(\zeta_{n}\right)$, and $\left|f_{n}^{j}\left(M_{n}\left(\zeta_{n}\right)\right)-M_{n}\left(\zeta_{n}\right)\right| \geq \varepsilon \rho_{n} / 2>0$ for $1 \leq j \leq k-1$. By (99), for $1 \leq j \leq k-1, f_{n}^{j}\left(M_{n}\left(\zeta_{n}\right)\right) \rightarrow z_{0} \in D$, as $n \rightarrow \infty$. It follows that for sufficiently large $n, M_{n}\left(\zeta_{n}\right)$ is a periodic point of period $k$ of $f_{n}$ in $D$. Since $f_{n}$ has no repelling periodic point of period $k$ in $D$, we have $\left|\left(f_{n}^{k}\right)^{\prime}\left(M_{n}\left(\zeta_{n}\right)\right)\right| \leq 1$, so that by $(98),\left|\left(H_{n}^{k}\right)^{\prime}\left(\zeta_{n}\right)\right| \leq 1$. Thus

$$
\left|\left(H^{k}\right)^{\prime}\left(\zeta_{0}\right)\right|=\left|\lim _{n \rightarrow \infty}\left(H_{n}^{k}\right)^{\prime}\left(\zeta_{n}\right)\right| \leq 1 .
$$

Claim 2 is proved.

By Claim 2 and Lemma 1, we see that $H$ must be a rational function. Write

$$
H(\zeta)=\zeta+c \frac{Q(\zeta)}{P(\zeta)}
$$

where $c \neq 0$ is a constant and where $P, Q$ are two monic co-prime polynomials. Set $p=\operatorname{deg}(P)$ and $q=\operatorname{deg}(Q)$. We claim

(i) $p \geq 1$;

(ii) $q \leq p+1$;

(iii) if $q=p+1$, then $|c+1|<1$;

(iv) if $q=p+1$ and $c=-1$, then $p=1$ and $q=2$. 
To prove (i), suppose $p=0$. Then $H$ is a polynomial. By Claims 1-2 and Lemma 15 with Remark 5, we have $\operatorname{deg}(H) \leq 1$. Thus $H(z)=a z+b$ for some constants $a, b$. By Claim 1, we see that $|a| \leq 1$. This contradicts $\frac{\left|H^{\prime}(0)-1\right|}{1+|H(0)|^{2}}=3$.

To prove (ii)-(iv), suppose that $q \geq p+1$. Let $r>\max \{|\zeta|: P(\zeta) Q(\zeta)=0\}$. Then, as $H_{n}(\zeta) \rightarrow H(\zeta)$, there are two monic co-prime polynomials $Q_{n}(\zeta)$ of degree $\operatorname{deg}\left(Q_{n}\right)=q$ and $P_{n}(\zeta)$ of degree $\operatorname{deg}\left(P_{n}\right)=p$ satisfying $Q_{n}(\zeta) \rightarrow Q(\zeta)$ and $P_{n}(\zeta) \rightarrow P(\zeta)$ such that

$$
H_{n}(\zeta)=\zeta+\frac{Q_{n}(\zeta)}{P_{n}(\zeta)} h_{n}(\zeta)
$$

where $h_{n}(\zeta) \rightarrow c$ uniformly on $\mathbb{C}$.

By assumption and (97), $H_{n}$ has no fixed point with multiplier 1 , so that all roots of $Q_{n}$ are simple. Thus

$$
Q_{n}(\zeta)=\left(\zeta-\zeta_{n, 1}\right)\left(\zeta-\zeta_{n, 2}\right) \cdots\left(\zeta-\zeta_{n, q}\right),
$$

where $\zeta_{n, j}$ are pairwise distinct and $\left|\zeta_{n, j}\right|<r$. We have

$$
\begin{aligned}
\sum_{j=1}^{q} \frac{1}{1-H_{n}^{\prime}\left(\zeta_{n, j}\right)} & =\sum_{j=1}^{q} \operatorname{Res}\left(\frac{1}{\zeta-H_{n}(\zeta)}, \zeta_{n, j}\right) \\
& =\frac{1}{2 \pi i} \int_{|\zeta|=r} \frac{\mathrm{d} \zeta}{\zeta-H_{n}(\zeta)} \\
& \rightarrow \frac{1}{2 \pi i} \int_{|\zeta|=r} \frac{\mathrm{d} \zeta}{\zeta-H(\zeta)} \\
& =-\operatorname{Res}\left(\frac{1}{\zeta-H(\zeta)}, \infty\right) \\
& = \begin{cases}-\frac{1}{c} & \text { if } q=p+1, \\
0 & \text { if } q \geq p+2 .\end{cases}
\end{aligned}
$$

However, a simple computation shows that $\left|H_{n}^{\prime}\left(\zeta_{n, j}\right)\right| \leq 1$ and $H_{n}^{\prime}\left(\zeta_{n, j}\right) \neq 1$ is equivalent to

$$
\operatorname{Re}\left(\frac{1}{1-H_{n}^{\prime}\left(\zeta_{n, j}\right)}\right) \geq \frac{1}{2} .
$$

By (107) and (108), we see that $q=p+1$ and

$$
\operatorname{Re}\left(-\frac{1}{c}\right) \geq \frac{q}{2}=\frac{p+1}{2} \geq 1 .
$$

It follows that $|c+1|<1$ and that if $c=-1$, then $p=1$ and $q=2$.

By (i)-(iv), $\infty$ is a weakly repelling fixed point of $H$ unless $\operatorname{deg}(H)=1$. The lemma is proved.

\section{Proofs of theorems AND COROLlaries}

Now we prove the results stated in the Introduction.

Proof of Theorem 1. Suppose that $\mathcal{F}$ is not normal at some point $z_{0} \in D$. Then by Lemma 17 , there exist points $z_{n} \in D$ with $z_{n} \rightarrow z_{0}$, functions $f_{n} \in \mathcal{F}$ and positive 
numbers $\rho_{n} \rightarrow 0$ such that

$$
H_{n}(\zeta)=\frac{f_{n}\left(z_{n}+\rho_{n} \zeta\right)-z_{n}}{\rho_{n}} \rightarrow H(\zeta)
$$

locally uniformly with respect to the spherical metric, where $H$ is a non-constant rational function, not of the form $\zeta+c$ with constant $c \in \mathbb{C}$, such that $H$ has no repelling periodic cycle of period $k$ in $\mathbb{C}$, and

$$
\frac{\left|H^{\prime}(0)-1\right|}{1+|H(0)|^{2}}=3 \text {. }
$$

We claim that all poles of $H$ have multiplicity $\geq 2$. Indeed, suppose that $H$ has a pole $\zeta_{0}$. Then there exists $\delta>0$ such that all $1 / H_{n}$ for sufficiently large $n$ and $1 / H$ are holomorphic on the disk $D_{\delta}\left(\zeta_{0}\right)=\left\{\left|\zeta-\zeta_{0}\right| \leq \delta\right\}$ and that $1 / H_{n} \rightarrow 1 / H$ uniformly on $D_{\delta}\left(\zeta_{0}\right)$.

Set1

$$
h_{n}(\zeta)=\frac{1}{H_{n}(\zeta)}-\frac{\rho_{n}}{a_{n}-z_{n}} \text { and } h(\zeta)=\frac{1}{H(\zeta)},
$$

where $a_{n}=a\left(f_{n}\right)$. Then $h_{n} \rightarrow h$ uniformly on $D_{\delta}\left(\zeta_{0}\right)$. Since $h\left(\zeta_{0}\right)=0$, by Hurwitz's Theorem, there exist points $\zeta_{n} \rightarrow \zeta_{0}$ such that $h_{n}\left(\zeta_{n}\right)=0$. Together with (110) and (112), this shows that $f_{n}\left(z_{n}+\rho_{n} \zeta_{n}\right)=a_{n}$. Since the $a_{n}$-points of $f_{n}$ have multiplicity $\geq 2$, we get $f_{n}^{\prime}\left(z_{n}+\rho_{n} \zeta_{n}\right)=0$, and since

$$
h_{n}^{\prime}(\zeta)=\frac{\rho_{n}^{2} f_{n}^{\prime}\left(z_{n}+\rho_{n} \zeta\right)}{\left[f_{n}\left(z_{n}+\rho_{n} \zeta\right)-z_{n}\right]^{2}},
$$

we have $h_{n}^{\prime}\left(\zeta_{n}\right)=0$ for sufficiently large $n$. Therefore,

$$
h^{\prime}\left(\zeta_{0}\right)=\lim _{n \rightarrow \infty} h_{n}^{\prime}\left(\zeta_{n}\right)=0 .
$$

It follows that $\zeta_{0}$ is a multiple zero of $h$ and thus a multiple pole of $H$.

Hence the poles of $H$ have multiplicity $\geq 2$.

Since affine conjugation preserves the multiplicity of the poles for rational functions, we see by Lemmas 4 and 5 that $\operatorname{deg}(H) \leq 1$.

Since the poles of $H$ have multiplicity $\geq 2$ and $\operatorname{deg}(H) \leq 1, H$ is analytic; hence $H(\zeta)=\alpha \zeta+\beta$ for some constants $\alpha$ and $\beta$. Since $H$ has no repelling fixed point in $\mathbb{C},|\alpha| \leq 1$, and thus $\frac{\left|H^{\prime}(0)-1\right|}{1+|H(0)|^{2}} \leq 2$. This contradicts (111).

The proofs of Theorems 3 and 5 are similar to that of Theorem 1, so we omit the details.

\section{REFERENCES}

[1] I. N. Baker, Fix-points of polynomials and rational functions, J. London Math. Soc. (2) 39 (1964), 615-622. MR0169989 (30:230)

[2] D. Bargmann and W. Bergweiler, Periodic points and normal families, Proc. Amer. Math. Soc 129 (2001), 2881-2888. MR.1840089 (2002c:30034)

[3] W. Bergweiler, Iteration of meromorphic functions, Bull. Amer. Math. Soc. (N. S.) 29 (1993), 151-188. MR1216719 (94c:30033)

[4] W. Bergweiler, Periodische Punkte bei der Iteration ganzer Funktionen, Habilitationsschrift, Rheinisch-Westfälische Techn. Hochsch., Aachen, 1991.

[5] W. Bergweiler, An Introduction to Complex Dynamics, Universidade de Coimbra, Coimbra, 1995. MR1626031 (99c:58133)

\footnotetext{
${ }^{1}$ If $a_{n}=\infty$, we consider $h_{n}(\zeta)=\frac{1}{H_{n}(\zeta)}$.
} 
[6] W. Bergweiler, Ahlfors theory and complex dynamics: Periodic points of entire functions, in "Complex dynamics and related fields", RIMS Kokyuroku 1269 (2002), 1-11. MR.1953889

[7] W. Bergweiler and N. Terglane, Weakly repulsive fixpoints and the connectivity of wandering domains, Trans. Amer. Math. Soc. 348 (1996), 1-12. MR.1327252 (96e:30055)

[8] L. Carleson and T. W. Gamelin, Complex Dynamics, Springer, 1993. MR.1230383 (94h:30033)

[9] J. M. Chang, Polynomials without repelling periodic points of given periods, J. Math. Anal. Appl. 324 (2006), 1-13. MR2262451 (2008a:37047)

[10] J. M. Chang, Normality, quasinormality and periodic points, Nagoya Math. J. 195 (2009), 77-95. MR 2552954 (2010h:37092)

[11] J. M. Chang and M. L. Fang, Normal families and fixed points, J. Anal. Math. 95 (2005), 389-395. MR2145570 (2005m:30034)

[12] J. M. Chang and M. L. Fang, Repelling periodic points of rational functions of given periods, Sci. China Ser. A 49 (2006), 1165-1174. MR2284204 (2007k:37055)

[13] J. M. Chang, M. L. Fang and L. Zalcman, Normality and attracting fixed points, Bull. London Math. Soc. 40 (2008), 777-788. MR2439643 (2009g:30038)

[14] M. Essén and S. J. Wu, Fix-points and normal families of analytic functions, Complex Variables Theory Appl. 37 (1998), 171-178. MR1687872 (99m:20069)

[15] M. Essén and S. J. Wu, Repulsive fixpoints of analytic functions with application to complex dynamics, J. London Math. Soc. (2) 62 (2000), 139-148. MR.1771857 (2001k:37065)

[16] W. K. Hayman, Meromorphic Functions, Clarendon Press, Oxford, 1964. MR0164038 (29:1337)

[17] J. Milnor, Dynamics in One Complex Variable, Princeton University Press, Princeton, 2006. MR2193309 (2006g:37070)

[18] X. C. Pang, Shared values and normal families, Analysis 22 (2002), 175-182. MR.1916423 (2003h:30043)

[19] X. C. Pang and L. Zalcman, Normal families and shared values, Bull. London Math. Soc. 32 (2000), 325-331. MR.1750485 (2001e:30059)

[20] J. Schiff, Normal Families, Springer-Verlag, 1993. MR1211641 (94f:30046)

[21] M. Shishikura, On the quasi-conformal surgery of rational functions, Ann. Sci. École Norm. Sup. (4) 20 (1987), 1-29. MR892140(88i:58099)

[22] N. Steinmetz, Rational Iteration, Walter de Gruyter, 1993. MR1224235 (94h:30035)

[23] L. Yang, Some recent results and problems in the theory of value distribution, Proceedings Symposium on Value Distribution Theory in Several Complex Variables (Notre Dame, IN, 1990) (W. Stoll, ed.), Univ. Notre Dame Press, 1992, pp. 157-171. MR.1243023 (94i:30029)

[24] L. Yang, Value Distribution Theory, Springer-Verlag, Berlin, 1993. MR,1301781 (95h:30039)

[25] L. Zalcman, A heuristic principle in complex function theory, Amer. Math. Monthly 82 (1975), 813-817. MR0379852 (52:757)

[26] L. Zalcman, Normal families: New perspectives, Bull. Amer. Math. Soc. (N. S.) 35 (1998), 215-230. MR:1624862 (99g:30048)

Department of Mathematics, Changshu Institute of Technology, Changshu, Jiangsu 215500, People's Republic of China

E-mail address: jmchang@cslg.edu.cn

Department of Mathematics, Bar-Ilan University, 52900 Ramat-Gan, Israel

E-mail address: zalcman@macs.biu.ac.il 